$09.1 ; 14$

\title{
Определение времен затухания и анизотропии поляризованной флуоресценции флавинадениндинуклеотида с субнаносекундным разрешением
}

\author{
(С) М.К. Краснопевцева, В.П. Белик, А.А. Богданов, И.В. Семенова, А.Г. Смолин, О.С. Васютинский \\ Физико-технический институт им. А.Ф. Иоффе РАН, Санкт-Петербург, Россия \\ E-mail: marina.krasnopevtceva@gmail.com
}

Поступило в Редакцию 7 февраля 2020г.

В окончательной редакции 7 февраля 2020 г.

Принято к публикации 27 марта 2020 г.

Исследованы оптические свойства биологического кофермента FAD (флавинадениндинуклеотид) в водном растворе. В экспериментах по наблюдению затухания поляризованной флуоресценции, возбуждаемой импульсами пикосекундного лазера, определены два времени жизни флуоресценции, время вращательной диффузии и параметр анизотропии. Проведено обсуждение полученных результатов и сравнение с результатами других авторов.

Ключевые слова: флавинадениндинуклеотид, FAD, поляризованная флуоресценция, время затухания, время вращательной диффузии, анизотропия.

DOI: 10.21883/PJTF.2020.12.49528.18234

Кофермент флавинадениндинуклеотид (FAD) является непосредственным участником окислительновосстановительных реакций в живых клетках. Флавинадениндинуклеотид существует в двух формах - окисленной FAD и восстановленной $\mathrm{FADH}_{2}$, причем $\mathrm{FADH}_{2}$ не флуоресцирует, а FAD обладает автофлуоресценцией и широко используется в качестве флуоресцентного зонда при исследовании биохимических процессов в живых клетках [1]. Известно, что параметры флуоресценции FAD меняются в зависимости от биохимических процессов, в которых он участвует $[1,2]$, поэтому определение этих параметров позволяет получить информацию об окислительно-восстановительных реакциях в живых клетках. FAD участвует главным образом в митохондриальных процессах, таких как бета-окисление жирных кислот и цикл Кребса, однако он присутствует также в ядре и цитозоле клеток [3]. Помимо этого FAD является кофактором фотолиазы - фермента репарации ДНК у прокариот, архей и некоторых эукариот [4], что дает возможность его использования в качестве флуоресцентного зонда в живых организмах. В настоящее время мониторинг параметров флуоресценции FAD используется в диагностике и терапии раковых заболеваний $[2,5]$. Большой интерес для исследования метаболизма в клетках представляет также сравнение излучения флуоресценции биологических коэнзимов FAD и NADH [6,7]. Известно, что в растворах FAD существует в двух конформациях: „открытой““, в которой его изоаллоксазиновая и адениновая хромоформные группы пространственно разделены, и „сложенной“, в которой между ими возникает химическая связь $\pi-\pi[8]$. Несмотря на проведенные исследования, имеющиеся в литературе данные о параметрах флуоресценции FAD в различных растворах неполны и зачастую противоречат друг другу $[8,9]$. Прежде всего это касается параметров затухания поляризованной флуоресценции при импульсном возбуждении, таких как времена жизни, отношение соответствующих весовых коэффициентов, времена вращательной диффузии, анизотропия поглощения.

В настоящей работе проведены исследования времен затухания поляризованной флуоресценции, отношения весовых коэффициентов, анизотропии поглощения и времени вращательной диффузии в водном растворе FAD для различных длин волн в пределах полосы флуоресценции.

В экспериментах использовался раствор FAD в дистиллированной воде $(\mathrm{pH}=5.7)$ с концентрацией $60 \mu \mathrm{M}$. На рис. 1, a представлен спектр поглощения водного раствора $\mathrm{FAD}$, зарегистрированный с помощью спектрофотометра Shimadzu UV-1800. Как видно из этого рисунка, в спектре поглощения FAD присутствуют два пика с максимумами на 375 и $450 \mathrm{~nm}$. Эти пики поглощения обычно интерпретируются как соответствующие оптическим переходам $S_{0} \rightarrow S_{2}$ и $S_{0} \rightarrow S_{1}$ во второе и первое электронные возбужденные состояния изоаллоксазиновой хромофорной группы FAD [8]. На рис. 1, $b$ приведен спектр флуоресценции, полученный при возбуждении молекул FAD полупроводниковым лазером на длине волны $452.5 \mathrm{~nm}$. Измерения осуществлялись с помощью монохроматора МДР-12 с дифракционной решеткой $1200 \mathrm{~mm}^{-1}$ и ширинами входной и выходной щелей $0.15 \mathrm{~mm}$, что соответствовало спектральному разрешению $0.35 \mathrm{~nm}$. Измерения проводились в диапазоне длин волн от 450 до $750 \mathrm{~nm}$, а регистрация излучения флуоресценции производилась фотоэлектронным умножителем (ФЭУ) Hamamatsu H10682-01. Небольшой пик на длине волны $452.5 \mathrm{~nm}$ на рис. $1, b$ обусловлен рассеянным излучением возбуждающего лазера. 

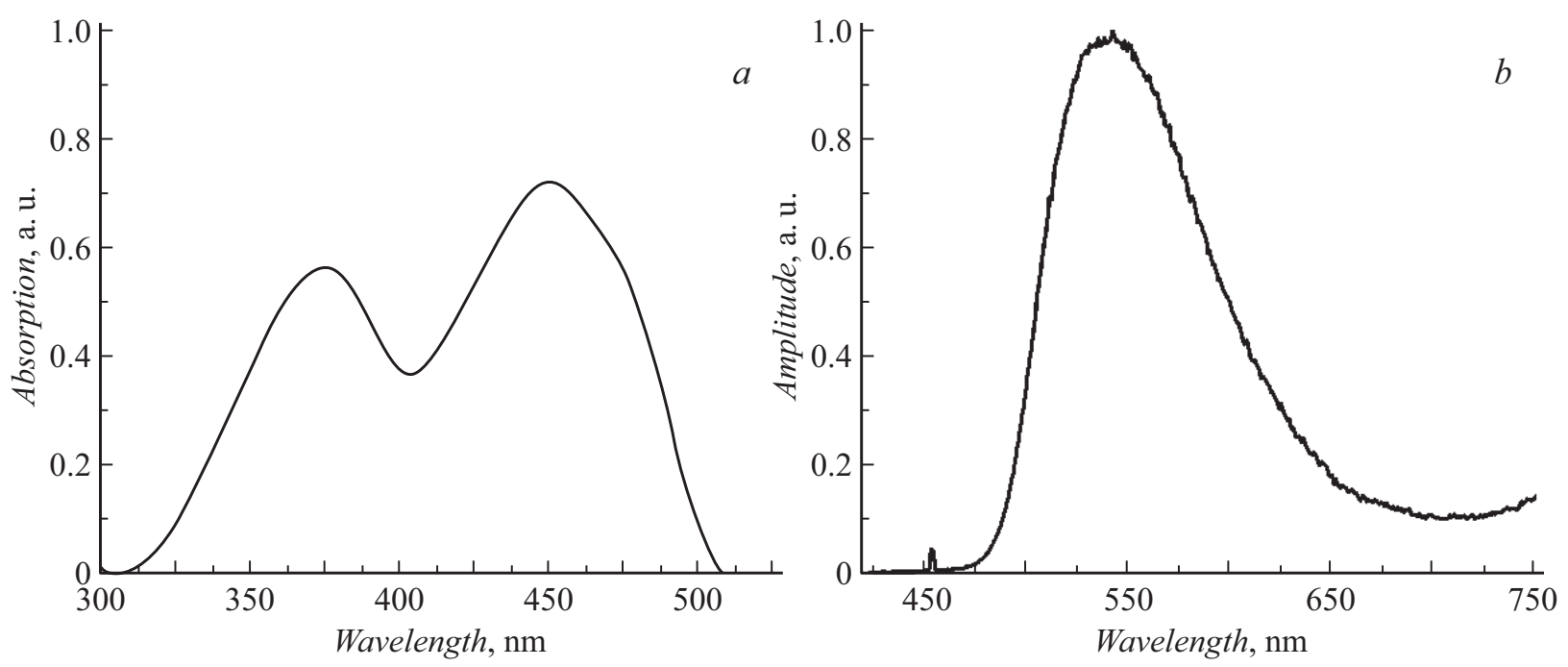

Рис. 1. Спектры поглощения $(a)$ и флуоресценции $(b)$ FAD в воде.

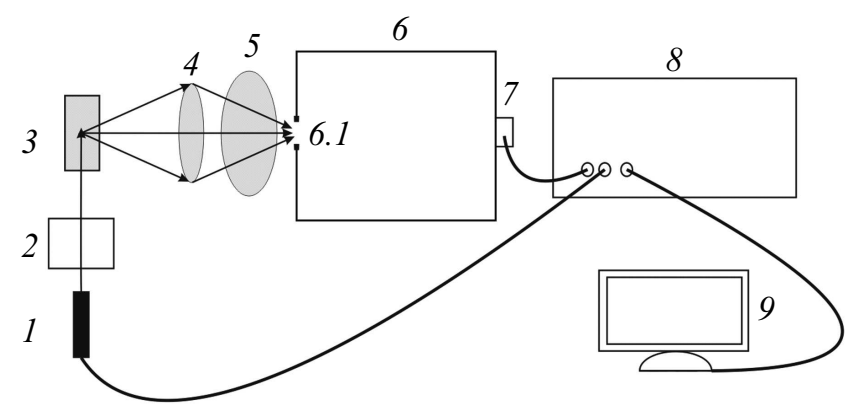

Рис. 2. Схема экпериментальной установки. 1 - лазер, $2-$ призма Глана, 3 - кювета с раствором, 4 - собирающая линза, 5 - поляризатор, 6 - монохрматор, 6.1- входная щель монохроматора, 7 - фотоумножитель, 8 - ТCSPC-модуль, 9 - компьютер.

Исследования затухания поляризованной флуоресценции FAD проводились в основном по методике, описанной в наших предыдущих работах [10-12], однако имелись и некоторые отличия. Схема установки представлена на рис. 2. Кварцевая кювета с водным раствором FAD $(10 \times 10 \mathrm{~mm}, 3.5 \mathrm{ml})$ облучалась импульсным полупроводниковым лазером на длине волны $452.5 \mathrm{~nm}$ с импульсами длительностью $30 \mathrm{ps}$ и частотой повторения $2 \mathrm{MHz}$. Лазерное излучение было поляризовано вертикально, параллельно входной щели монохроматора (ось $Y$ ). Излучение флуоресценции раствора фокусировалось на входную щель монохроматора МДР-12 под прямым углом к направлению лазерного пучка. Измерения проводились на длинах волн $\lambda=510,520,530$, 550 и $570 \mathrm{~nm}$ в пределах полосы флуоресценции FAD. Непосредственно перед входной щелью монохроматора был помещен тонкопленочный дихроичный поляризатор, ось пропускания которого поочередно устанавливалась в вертикальное и горизонтальное положение. Регистрация временно́й динамики затухания поляризованной флуоресценции раствора на выходе из монохроматора осуществлялась с помощью ФЭУ Hamamatsu H10682-01 при двух взаимно перпендикулярных положениях оси поляризатора. Детектирование сигналов флуоресценции осуществлялось в режиме счета фотонов, а их последующая обработка производилась по методу времякорреляционной спектроскопии (ТCSPC) с помощью модуля PicoHarp 300 (PicoQuant).

На рис. 3 представлены типичные кривые затухания поляризованной флуоресценции FAD на длине волны $510 \mathrm{~nm}$. Символами обозначены полученные экспериментальные данные, а сплошными кривыми - результаты подгонки. $I_{x}$ и $I_{y}$ на рис. 3 обозначают интенсивности горизонтально и вертикально поляризованных компонент флуоресценции, a IRF — график аппаратной функции использованного измерительного тракта, полуширина которого составляла 320 ps.

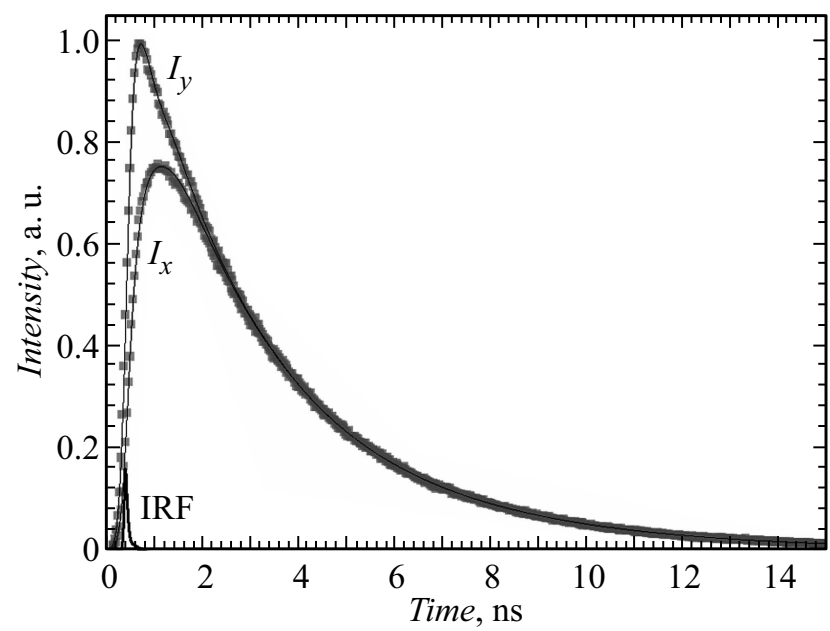

Pис. 3. Сигналы затухания поляризованной флуоресценции. Время $t=0$ соответствует моменту возбуждения. 
Параметры флуоресценции

\begin{tabular}{c|c|c|c|c|c}
\hline$\lambda, \mathrm{nm}$ & $\tau_{1}, \mathrm{~ns}$ & $\tau_{2}, \mathrm{~ns}$ & $I_{1} / I_{2}$ & $r_{0}$ & $\tau_{\text {rot }}, \mathrm{ns}$ \\
\hline 510 & $4.3 \pm 0.2$ & $2.2 \pm 0.2$ & $0.58 \pm 0.06$ & $0.34 \pm 0.03$ & $0.27 \pm 0.03$ \\
520 & $4.2 \pm 0.2$ & $2.1 \pm 0.2$ & $0.61 \pm 0.06$ & $0.35 \pm 0.03$ & $0.24 \pm 0.03$ \\
530 & $4.2 \pm 0.2$ & $2.1 \pm 0.2$ & $0.62 \pm 0.06$ & $0.35 \pm 0.03$ & $0.24 \pm 0.03$ \\
550 & $4.2 \pm 0.2$ & $2.2 \pm 0.2$ & $0.63 \pm 0.06$ & $0.35 \pm 0.03$ & $0.26 \pm 0.03$ \\
570 & $4.2 \pm 0.2$ & $2.1 \pm 0.2$ & $0.60 \pm 0.06$ & $0.33 \pm 0.03$ & $0.27 \pm 0.03$
\end{tabular}

Как известно, если поляризация возбуждающего излучения направлена вдоль оси $Y$, сигналы затухания поляризованной флуоресценции, распространяющейся вдоль оси $Z$, могут быть описаны следующими выражениями [13]:

$$
\begin{gathered}
I_{x}=I_{0}(t)[1-r(t)], \\
I_{y}=G I_{0}(t)[1+2 r(t)],
\end{gathered}
$$

где индексы $x$ и $y$ задают соответствующие компоненты поляризации флуоресценции, $I_{0}(t)-$ изотропная компонента излучения флуоресценции, $G$ - отношение чувствительности в $x$ - и $y$-каналах регистрации, а $r(t)-$ параметр анизотропии.

Полученные экспериментальные данные обрабатывались на основе выражений (1), (2) с помощью процедуры глобальной подгонки, реализованной в среде MATLAB, в рамках которой осуществлялась свертка выражений (1) и (2) с аппаратным контуром $\operatorname{IRF}(t)$, приведенным на рис. 3. В результате было установлено, что временна́я зависимость изотропной компоненты излучения флуоресценции $I_{0}(t)$ в условиях наших экспериментов удовлетворительно описывалась двухэкспоненциальной функцией

$$
I_{0}(t)=\left[I_{1} \exp \left(-t / \tau_{1}\right)+I_{2} \exp \left(-t / \tau_{2}\right)\right]
$$

где $I_{1}$ и $I_{2}-$ предэкспоненциальные коэффициенты, а $\tau_{1}, \tau_{2}$ - времена затухания флуоресценции.

Параметр анизотропии $r(t)$ описывался одноэкспоненциальной функцией

$$
r(t)=r_{0} \exp \left(-t / \tau_{\text {rot }}\right)
$$

где $r_{0}$ - анизотропия в момент возбуждения, а $\tau_{r o t}-$ время вращательной диффузии.

Результаты подгонки представлены в виде сплошных кривых на рис. 3, а полученные параметры для нескольких длин волн флуоресценции приведены в таблице. Видно, что эти параметры не зависят от длины волны флуоресценции в пределах погрешностей. Результаты статистического анализа экспериментальных данных, приведенные в таблице, свидетельствуют о наличии двух времен жизни в возбужденном состоянии FAD, что подтверждает результаты работы [9], но не согласуется с выводами работы [8], где сообщалось о наличии четырех времен жизни при тех же экспериментальных условиях. Средние значения приведенных в таблице времен жизни $\tau_{1}=4.2 \pm 0.2 \mathrm{~ns}$ и $\tau_{2}=2.1 \pm 0.2 \mathrm{~ns}$ в пределах погрешностей совпадают с полученными в работе [9] в водном растворе FAD, однако обоснованность проведенного в [9] усреднения этих двух времен не подтверждается нашим анализом. Вероятно, время $\tau_{1}$ соответствует времени жизни FAD в открытой конформации, поскольку его значение близко к времени жизни свободного флавина [14], а время $\tau_{2}$ отражает наличие в растворе молекул FAD в сложенной конформации. Определенный в настоящей работе параметр анизотропии $r_{0}=0.34 \pm 0.03$ близок к его максимальному значению для симметричного волчка $\left(r_{\max }=0.4\right)$, что свидетельствует о том, что дипольные моменты возбуждения и флуоресценции FAD в воде приблизительно параллельны друг другу.

\section{Благодарности}

Авторы благодарят ФТИ им. А.Ф. Иоффе за предоставление оборудования для проведения экспериментов.

\section{Финансирование работы}

Работа выполнена при поддержке Российского фонда фундаментальных исследований (грант № 18-53-34001).

\section{Конфликт интересов}

Авторы заявляют, что у них нет конфликта интересов.

\section{Список литературы}

[1] Galbán J., Sanz-Vicente I., Navarro J., de Marcos S. // Methods Appl. Fluoresc. 2016. V. 4. P. 042005.

[2] Pradhan A., Pandey P.K., Singh P. Overview of fluorescence spectroscopy in early cancer detection // Neurophotonics and biomedical spectroscopy / Eds R.R. Alfano, L. Shi. Amsterdam-Oxford-Cambridge: Elsevier, 2019. P. 253-328.

[3] Giancaspero T.A., Busco G., Panebianco C., Carmone C., Miccolis A., Liuzzi G.M., Colella M., Barile M. // J. Biol. Chem. 2013. V. 288. P. 29069-29080.

[4] Müller P., Brettel K., Grama L., Nyitrai M., Lukacs A. // ChemPhysChem. 2016. V. 17. P. 1329-1340.

[5] Shirmanova M.V., Druzhkova I.N., Lukina M.M., Dudenkova V.V., Ignatova N.I., Snopova L.B., Shcheslavskiy V.I., Belousov V.V., Zagaynova E.V. // Sci. Rep. 2017. V. 7. P. 8911. 
[6] Wall M.A., Heaster T.M., Tilbury K., Choi W.J., Roblyer D., Wang R., Skala M., Liu J.T.C. Metabolic imaging approaches: optical imaging // Imaging and metabolism / Eds J.S. Lewis, K.R. Keshari. N.Y.: Springer, 2018. P. 113.

[7] Васютинский O.C., Смолин А.Г., Oswald C., Gericke K.-H. // Оптика и спектроскопия. 2017. Т. 122. B. 4. C. $622-626$.

[8] Nakabayashi T., Islam M.S., Ohta N. // J. Phys. Chem. B. 2010. V. 114. P 15254-15260.

[9] Sengupta A., Khade R.V., Hazra P. // J. Photochem. Photobiol. 2011. V. 221. P 105-112.

[10] Sasin M.E., Smolin A.G., Gericke K.-H., Tokunaga E., Vasyutinskii O.S. // Phys. Chem. Chem. Phys. 2018. V. 20. P. 19922-19931.

[11] Denicke S., Gericke K.-H., Smolin A.G., Shternin P.S., Vasyutinskii O.S. // J. Phys. Chem. A. 2010. V. 114. P. 96819692.

[12] Herbrich S., Al-Hadhuri T., Gericke K.-H., Shternin P.S., Smolin A.G., Vasyutinskii O.S. // J. Chem. Phys. 2015. V. 142. P. 024310.

[13] Shternin P.S., Gericke K.-H., Vasyutinskii O.S. // Mol. Phys. 2010. V. 108. P 813-825.

[14] Drossler P., Holzer H., Penzkofer A., Hegemann P. // Chem. Phys. 2002. V. 282. P. 429-439. 